\title{
The Influence of New Media on Feminist Movement: An Analysis of Feminist Images on Weibo
}

\author{
Wanjing $\mathrm{Gu}^{1, \dagger}$ Jiaxi Jiang ${ }^{2, *, \dagger}$ and $\mathrm{Zi} \mathrm{Ye}^{3, \dagger}$ \\ ${ }^{1}$ Faculty of Health, York University, North York, M2N 0L1, Canada \\ ${ }^{2}$ College of Art and Science, University of Washington, Seattle, 98105, United States \\ ${ }^{3}$ School of Humanities, University of California, Irvine, 92606, United States \\ These authors contributed equally. \\ ${ }^{*}$ Corresponding author. Email: jxjiang@uw.edu
}

\begin{abstract}
Weibo has been the most commonly used platform for Chinese people to speak out in the feminist movement in recent years. Therefore, our group plans to conduct a more in-depth analysis and research on the images of feminist activities on Weibo to reflect the influence of media. This paper mainly discusses the influence of Weibo on China's feminist movement compared with traditional media. The purpose is to understand people's cognition and understanding of the feminist movement by investigating the influence of media on the feminist movement. At the same time, this paper will discuss the influence of new and old media on social events, as well as the influence of new media on women's events and the feminist movement through this research. Further, this paper will discuss the advantages of new media in publicizing social events compared with traditional media in China. In conclusion, new media, such as microblogs, have had a positive effect on the feminist movement by giving more people a voice, allowing women to defend their rights, and making more people aware of the feminist movement.
\end{abstract}

Keywords: New media, Feminist, Weibo.

\section{INTRODUCTION}

Coverage of COVID-19 in Mainstream Chinese media, such as TV shows and state-run media, has often been criticized for the lack of female representation, which is a kind of neglect of women's hard work in the fight against the epidemic. Feminists reacted angrily on Chinese social media Weibo [1]. Weibo refers to a broadcast social media and network platform based on user relationship information sharing, dissemination, and acquisition, which shares short real-time information through the following mechanism. It is a kind of social network connection with content as the link. Its communication structure is "personal canter" + "content connection". Meanwhile, Weibo is the most popular and most widely used media platform in China besides Wechat. The biggest advantage of Weibo is that it not only spreads information very fast but also gives the public a space to express their opinions freely.

Feminism is a range of social movements, political movements, and ideologies that aim to define and establish the political, economic, personal, and social equality of the sexes. Feminism in China, in particular, has a very different cultural background and characteristics. There is an ancient myth in China that indicates the beginning of feminism: The legend of NvWa. It tells us that women have had an important role in Chinese society since the beginning of time [2]. However, the idea of "men are superior to women" has been carried out later on in the long history of China. This makes feminism extremely difficult to promote in China. It was not until the New Culture Movement and the May 4th Movement in the last century that the female group and some distinct female images were gradually brought into the public's view. When we shift to the present, the emergence of a large number of social media gives rise to the phenomenon of "everyone is wemedia", and audiences have the opportunity to express themselves. This empowerment changes the status quo of women in the media, and more and more women find groups to jointly speak for their rights and interests. When women come into contact with feminist remarks on the Internet, they are no longer satisfied with the status of being stared at by men, and they begin to rebel against patriarchy. Many women participate in public 
discussions and fight for the pursuit of self-worth and equal rights.

The development of the Internet has provided the public with a free space for public opinion. Feminism has been widely spread in this free space. More and more women choose to become feminists and speak out as feminists in the media. With the emergence of Weibo, in particular, it is more convenient for feminists to join like-minded groups and participate in the propaganda process of feminist values, resulting in the rapid development of feminism. The convenient Internet allows feminists to use new media with men freely, to rebel against the patriarchal society through the channel of new media, and to vent their emotions that they cannot express in real life on the Internet. These media groups stand out as key voices in Chinese feminist and youth activism today, with implications for how we understand contemporary feminism on an international scale [3].

Weibo is the most commonly used platform for Chinese people to make their voices heard on feminist activities in recent years. Therefore, our group plans to conduct a deeper analysis and research on the image of feminist activities on Weibo, so as to reflect the influence of media. This paper focuses on exploring the impact of Weibo on the feminist movement in China compared to traditional media. The purpose is to understand people's perceptions and understanding of feminism by investigating the influence of the media on the movement. Meanwhile, this paper will investigate the influence of old and new media on social events and the influence of new media on women's events and the feminist movement through this research. Furthermore, this paper will explore what are the advantages of new media in publicizing social events compared with traditional media in China.

In addition to exploring the influence of media, this paper hopes to have an impact on the publicity of feminism. It is hoped that the society can correctly understand and apply the influence of women's rights in new media through this paper. This will play an important role in promoting the process of China's feminist movement.

\section{LITERATURE REVIEW}

\subsection{Old Media Versus NeW Media In ChINa}

The development and difference between new and old media largely determine the trend of feminist movement with Chinese characteristics. They determine the trend of feminist movement with Chinese characteristics. There is a literature on CNKI that analyzes the difference between new and old media and the characteristics of the two media. Cong Ren, the author, stated that "in the traditional media, such as newspapers, magazines, radio and television, the media present the image of feminists to the audience according to their own reporting position and frame. Feminists can only passively accept the image building." While in the new media era, websites on the Internet have shifted their coverage from male to female in an effort to attract more clicks. All kinds of women have become the main characters in online news. Especially during Web 2.0time, information technology has been further developed. Advances in mobile communication technology have made it possible for people to express their opinions at any time [2].

\subsection{FORMER LITERATURE REVIEW ABOUT FEMINISM IMAGE}

At present, there is a large amount of literature on the Internet to analyse the image of women's rights on Weibo. Most of the articles are analysed from the perspective of Weibo's platform nature and feminism with Chinese characteristics. When reviewing the history of the development of women's rights in China, patriarchy dominated most of the time. The development of feminism in China is narrow and difficult [3]. However, according to Holly Hou, through Weibo and other social media, grass-roots feminists have opened up a new bottom-up mode of activism different from the dominant top-down paradigm prevalent since the 1980s [4]. Chinese feminism has made great progress toward gender equality, which can be shown through people's perspectives on social media. Mengmeng Liu analysed two significant cases on Weibo and found out that, despite the potential for Weibo to promote different messages in terms of gender equality, people's reactions on these two cases reveal the development of Chinese feminism in contemporary China [5].

Some papers link feminism to social concerns. According to Siban Li's article "The Past and Present Life of Microblogging Feminism: From 'Politically Correct' to 'Commercially Correct'", people can understand how the proponents of the feminist movement are campaigning for a public [6]. There is a new term brought up by a group of scholars from Nanjing, China: Femvertising, which aims at winning more female customers by promoting female power [7]. There are also studies that show that, from a unique Chinese context, the growth of feminism in China shows progress, specifically because Chinese women have started to push more assertively for equality. Therefore, Chinese women indeed have broadened their use of modern information technology. As a medium of expression, social media platforms like Weibo have received the attention of an increasing number of female users [8]. However, these studies also coincidentally point out that feminism has not received enough 
attention in society at present. There are still some misleading and deliberately off-rhythm phenomena.

These studies and theories above help us understand some background information in advance. However, there still exist some research gaps. While much of the literature has analysed the image of feminists on Weibo, very little has been done to show exactly what effect Weibo can have on promoting feminism. In addition, most literature only compares the old and new media in a broad sense, and does not compare Weibo with traditional media alone. There is no literature to show what are the benefits and highlights of Weibo as a promotion of women's image and feminist movement. We hope that through our research and the analysis of existing literature, we can further expand the cognition of Weibo feminist image from the perspective of Chinese history and culture. This paper will use literature analysis method and in-depth interview method to conduct the research.

\section{Methodology}

The methodology for this paper was interviewing. The researchers found two people who were active in both social media and feminism to question. Both interviewees work in new media-related professions and have a basic understanding of how the Internet works, and both have studied abroad in North America, so they have some knowledge of the development of the feminist movement. The two interviewees had a consistent presence on social media, and gradually their accounts had gained followers. The researcher held a one-hour online meeting with the interviewees on WeChat. The interviewees expressed their views on some feminist movements on Weibo. For example, what are the similarities and differences between feminist issues in North America and feminist issues in China, and why there are big differences between men's and women's understanding of feminist issues. How do people of different age groups understand the feminist movement? Will the voices of public figures lead to a deeper understanding of the feminist movement? They also analyse the problems of the feminist movement in China from the perspectives of history, culture, and public opinion, and the solutions to these problems. In both interviews, the interviewees were more cooperative than expected, and they even said things that the researchers had not thought of before, which made the whole paper more comprehensive. One of the interviewees is now working in counselling, so she provided the researcher with many examples of inequalities that women encounter at work and at home during the interview. She believes that China still has a long way to go to protect women's rights. So, this article uses the interviews to add more detail to the topic of the study, and more ways of looking at the issue. The researcher analyses the similarities and differences between the views of the two interviewees, and their views are representative of the views of some Chinese women on gender equality nowadays.

\section{RESULTS AND DISCUSSION}

After the interviews and content analysis for this research, the collected text and data represent that in China, New media (Weibo) has a larger influence on feminist activities compared to traditional media. We will address three results that we found from our interviews and content analysis to analyse and discuss the reason for the result, the positive or negative effect that the results bring to the feminist movement in China, and the solutions or advice for them.

\subsection{WeIbo As A PlatForm}

Weibo provides a platform for disadvantaged groups to make their voices heard. In our interviews, we heard words like "vulnerable groups" and "voices" repeatedly from influential women's rights users on Weibo. This is of great and profound significance to the influence of feminism on the feminist movement in China's social media -- Weibo. Feminism as part of the underprivileged; From not being heard before, to speaking on Weibo can let many people know what feminism is and feminist ideas and consciousness. This means that the influence of the Weibo platform on the spread of feminism cannot be underestimated. As a voice platform, Weibo allows the public to publish unfair events on the public platform. As one of the emerging feminist forces, Weibo feminism can use Weibo to publish gender inequality, gender discrimination, and other events on Weibo to attract more people's attention. At the same time, the negative impact of Weibo on feminism as a voice platform cannot be ignored. Because social media such as Weibo do not require real-name authentication, people are free to fabricate facts under the protection of the Internet. A lot of feminism on Weibo is fake feminism, their real aim is to stir up gender quarrels and stigmatize feminism. Weibo should continue to strengthen its function as a voice platform for the underclass, which can help many people who experience inequality in society. Because Weibo is the only place for them to fight for their rights. The opacity of social media is an unavoidable problem. People use their own nicknames on the Internet, and they can set their gender as they like. Gender has become more of a focus in such gender-sensitive issues.

\subsection{WeIBo AS A TOOL FOR WOMEN}

Women, especially, can use social media as a "weapon" to defend their rights. In the interview, sexual harassment, sexual assault, and other words were also mentioned by the interviewees many times. They also 
pointed to the \# MeToo movement that has recently become widely known on China's Weibo social media platform. Many women who have been sexually assaulted no longer worry about being criticized for sharing their stories and experiences on social networks. They have the courage to publish their experiences to society and use Weibo as a "weapon" to protect their rights. There are clearly positive aspects to Weibo's influence in this regard. Many \# girls help girls, \# Stop Body shame started to become more and more popular in China. Because of the existence of Weibo, these factors that can make women feel insecure can be eliminated. More and more women are using Twitter as a weapon to defend their gender equality, body, reproductive rights, maternity leave, sexual violence, and other issues. The function of Weibo as a weapon should not be restricted or restricted by anyone. Weibo should protect women who publish their experiences and make sure they are not harmed again.

\subsection{WEIBO AS A NEW MEDIA}

After analyzing Weibo for the feminist movement, Weibo, as a new media, has a lot of functions related to information like other media does. Unlike traditional media, Weibo, as a new medium, provides a platform for every user to share their opinions. Just like the slogan of Weibo: "Post new news anytime and anywhere", every user can express their ideas and views anytime and anywhere on Weibo. It is such functionality that enables more information to be spread at a faster speed and in a wider range on Weibo. In fact, the new media, whether it promotes women's rights movement or other social events, has very significant advantages. It can spread in a fast range, so it can get certain events to more people; it can spread more widely, so the whole world can get to know what is happening in a moment. New media can make a particular value known to more people, but it depends on whether it is correct or diversified. At the same time, such rapid dissemination is easy to be distracted. The rapid dissemination of self, media, or new media makes it difficult for people to focus on one thing for a long time. The empathy ability and the power of expression will be degraded. People will rather be in the audience than to participate in public communication. This will let the voices of those who already have some other attempts, or have negative emotions amplified. New media sometimes will promote or incite some offensive words, but not adequate enough to communicate and understand. New media will highlight a lot of extreme viewpoints because it needs to be quick and eye-catching in combination with current affairs.

Therefore, for new media, everyone is different. Emphasizing the difference will cause more and more people to pay attention to their views and persuade others. In that case, everyone is to see a different side instead of seeing the similarity and empathy. The solution of this part of Weibo is that we cannot give advice or answers. These are the features of Weibo as a new media platform.

Weibo generates a lot of positive effects on the feminist movement in China and contributes to the completion of the women's image on Weibo as well. These features of Weibo as a new media application help the Chinese better understand the meaning of feminism and make feminism become a popular word in Chinese media. The fundamental reason Weibo can benefit the feminist movement is that a large population uses it in China. So Weibo's function as a media integrated with social ability positively affects the feminism movement in China, gaining more and more attention and focus.

\section{CONClusion}

Because of the instantaneous, interactive, timesensitive, and distorted nature of the new media, it has created a highly publicized and rapidly growing feminist movement in China. More people recognize the importance of gender equality and start to speak out for women. So, in this thesis, the researcher examines the impact of new media and on media on the feminist movement, the analysis of the images of the feminist movement on Weibo, and the impact of these images. After the researcher analyzed the content of the interviews, they got three results about the impact of new media on the feminist movement, which are: giving more people a voice, allowing women to defend their rights, and making more people aware of the feminist movement. First of all, Weibo provides a platform for people to express their views freely, and people can express any views they want on Weibo, without any distinction of right or wrong. This has also made it easier for some of the pioneers of the feminist movement to educate more people about what feminism is and how to protect themselves when women are in danger or are treated unfairly. However, there are times when some people use features such as Weibo's non-real names to deliberately fabricate facts in order to gain more attention and discredit feminism. Secondly, Weibo can also be used as a "weapon" for people to defend themselves, such as the MeToo movement and Girls help Girls, which allows more women to legally defend their basic rights and gives women a greater sense of security. Thirdly, with the rapid development of new media, more and more people are using microblogs and other software, which means that information on microblogs can be quickly disseminated to many people, and many issues that are not voiced offline can be voiced online. This means that information on Weibo can be quickly disseminated to many people. Many issues that are not voiced offline can be voiced online. In short, new media, such as microblogs, have had a 
positive effect on the feminist movement. At the same time it is also hoped that this article will make more people aware of the development model and channels of feminist movement in China. It is hoped that more people will learn about feminist movements and that in the future researchers will focus more on the trajectory and focus of feminist movements in different cultural contexts, as the focus of feminist movements in each country will be different depending on the cultural context. It is hoped that cultural differences and cultural inclusiveness can be compared and contrasted.

\section{REFERENCES}

[1] Zhang, J., Feminist responses on weibo aim to fight the misrepresentation of women during COVID-19 in China. The Conversation. Retrieved October 21, 2021, from https://theconversation.com/feministresponses-on-weibo-aim-to-fight-the-mi srepresentation-of-women-during-covid-19-inchina-169341, (2021).

[2] Menke, A., The development of feminism in China University of Montana (2017).

[3] Wang, B., Chinese feminists on social media: Articulating different voices, building strategic alliances. Chinese feminists on social media: articulating different voices, building strategic alliances (2018).

[4] Ren, C., Exploring the media image of feminists in the microblogging platform, (2021).

[5] Wang, Q., Women do not fight for their own rights, will men give you? WeChat public platform. Retrieved October 13, 2021, from https://mp.wei xin.qq.com/s/o-4N5cudACyv28ZIsZwgVg, (2021).

[6] HOLLY LIXIAN HOU, On Fire in Weibo: Feminist Online Activism in China, Economic and Political Weekly, Vol. 50, No. 17, pp. 79-85, (2015).

[7] Liu, M., The development of Chinese feminism on weibo. The Development of Chinese Feminism on Weibo, (2016).

[8] Li, S., The past life and present life of microblogging feminism: from "political correctness" to "commercial correctness". Retrieved October 13, 2021, from https://user.guancha.cn/main/content?id=330877, (2020)

[9] Chen, Y., Zhang, Z., \& Xia, Z., Sentiment assessment of brand advertising on gender issues on social network: A case study of femvertising on Sina Weibo in China. Sentiment Assessment of Brand Advertising on Gender Issues on Social Network: A Case Study of Femvertising on Sina Weibo in China, (2021).
[10] Lu, Y., Social Media Technology and female emancipation in China: Case study in sina-weibo. Social Media Technology and Female Emancipation in China: Case Study in Sina-Weibo, (2016). 